\title{
Left temporal lobe structural and functional abnormality underlying auditory hallucinations in schizophrenia
}

\author{
Kenneth Hugdahl ${ }^{1,2}$ *, Else-Marie Løberg ${ }^{1,2}$ and Merethe Nygård ${ }^{1}$ \\ 1 Department of Biological and Medical Psychology, University of Bergen, Bergen, Norway \\ 2 Division of Psychiatry, Haukeland University Hospital, Bergen, Norway
}

Edited by:

Francisco Barceló, University

of Illes Balears, Spain

Reviewed by:

Tsuyoshi Miyakawa, Kyoto University,

Graduate School of Medicine, Japan;

Fujita Health University, Japan

Francisco Barcelo, University

of Illes Balears, Spain

* Correspondence:

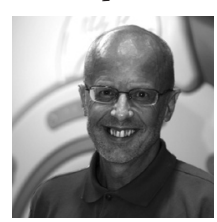

Kenneth Hugdahl is Professor of Biological Psychology at the University of Bergen, Norway and Adjunct Researcher at the Division of Psychiatry, Haukeland University Hospital, Bergen. He is the Head of the Bergen fMRI-group, which is a node in the Nordic Center of Excellence in Cognitive Control. His main research interests are in cognitive neuroscience, using $f M R I$ and other measures of brain function, e.g., dichotic listening, to elucidate questions related to hemispheric asymmetry.

In recent years he has applied this research to the understanding of auditory hallucinations in schizophrenia. Hugdahl@psybp.uib.no

In this article, we have reviewed recent findings from our laboratory, originally presented in Hugdahl et al. (2008). These findings reveal that auditory hallucinations in schizophrenia should best be conceptualized as internally generated speech mis-representations lateralized to the left superior temporal gyrus and sulcus, not cognitively suppressed due to enhanced attention to the 'voices' and failure of fronto-parietal executive control functions. An overview of diagnostic questionnaires for scoring of symptoms is presented together with a review of behavioral, structural, and functional MRI data. Functional imaging data have either shown increased or decreased activation depending on whether patients have been presented an external stimulus during scanning. Structural imaging data have shown reduction of grey matter density and volume in the same areas in the temporal lobe. We have proposed a model for the understanding of auditory hallucinations that trace the origin of auditory hallucinations to neuronal abnormality in the speech areas in the left temporal lobe, which is not suppressed by volitional cognitive control processes, due to dysfunctional fronto-parietal executive cortical networks.

Keywords: auditory hallucinations, schizophrenia, positive symptoms, dichotic listening, fMRI

\section{HALLUCINATIONS IN SCHIZOPHRENIA}

Hallucinations occur in $70-80 \%$ of patients with schizophrenia and often produce distress, functional disability, and behavioral dys-control (cf. Shergill et al., 1998). In some cases, hallucinations may also pose a threat to the patient or his/ her family members, and even to the society, if it takes the form of 'hearing voices' commanding the patient to commit unwanted behaviors or acts. The DSM-IV diagnostic manual for mental disorders (American Psychiatric Association, 1994) notes that 'hearing voices' is sufficient for a diagnosis of schizophrenia if 'the hallucinations consist of a voice keeping up a running commentary on the person's behaviour or two or more voices conversing with each other' (p. 285, note). Also, it should be noted that the voices should have been present for more than 6 months. An auditory halucintion can therefore be seen as a key symptom to diagnose schizophrenia.

Hallucinatory-like experiences are a core symptom in schizophrenia. Such experiences are also seen to occur among healthy population with a life-time prevalence of approximately $4-5 \%$ (Krabbendam and van Os, 2005; Tien, 1991). An important distinction between psychotic and non-psychotic hallucinations is the attribution of the source of the voices. Non-psychotic individuals typically attribute their experiences to internal sources, while psychotic patients often attribute their experiences to external sources. Hence, to qualify as a symptom, hallucinations are typically attributed to coming from outside of the person. 
Auditory hallucinations Auditory hallucinations in schizophrenia are seen as perceptual mis-representations.
Experiencing a hallucination as 'someone speaking to me' would in addition mean a lack of reality orientation, as often observed in patients with schizophrenia. The voices experienced by patients with schizophrenia often have an emotional and negative content, like 'You are worthless, and could as well be dead', or could take the form of imperative statements like 'Get lost, you should not be here'. It will however be interesting to find out if the 'voices' heard by non-psychotic individuals share cognitive and/or neuronal symptoms with individuals diagnosed with schizophrenia. Such an overlap would have consequences for current theories and models of schizophrenia in general, particularly for diagnostic categorization in psychiatry. If hallucinating individuals without a history of psychosis show similar behavioral and brain abnormalities as hallucinating individuals with schizophrenia, this would inform on the existence of a 'hallucination' factor, independent of disease pathology and diagnostic category.

\section{DIAGNOSTIC AND SYMPTOM SCALES}

Frequency and duration of hallucinations are typically scored from symptom scales like the Brief Psychiatric Rating Scale (BPRS; Ventura et al., 1993), the Positive and Negative Symptom Scale (PANSS; Kay et al., 1987) and Scales for Assessment of Negative and Positive Symptoms (SANS/SAPS; Andreasen, 1990). Positive symptoms are unwanted thoughts and behaviors that are in excess and out of proportion of what could be considered natural or normal, for e.g., hallucinations, delusions and overt conversation with a non-existing person. Treatment for positive symptoms can help in reducing the frequency and intensity of such thoughts and behaviors.

Negative symptoms, on the other hand, are behaviors that should be increased, e.g., passivity and lack of social interaction, as well as emotional blunting and lack of extrovert behaviors. Treatment for negative symptoms is therefore aimed at increasing what is lacking rather than reducing what is in excess, as in the case of positive symptoms.

The BPRS, PANSS, and SANS/SAPS scales contain statements regarding the experience of specific symptoms that are scored according to frequency and intensity. For example, the BPRS scale contains items indicating whether the patient shows signs of 'confused thought processes that are disconnected, disorganized, or disrupted', or if the patient shows 'exaggerated self-opinion, conviction, or unusual power or abilities'. The PANSS scale has items like 'verbal report or behavior indicating perceptions which are not generated by external stimuli', or 'diminished emotional responsiveness as characterized by reduction in facial expression, modulation of feelings, and communicative gestures'. The SAPS contains items like 'Have you ever heard voices and sounds when no one is around', and 'What did they say'. Currently, the different scales could be regarded as more or less interchangeable, when it comes to intra-class correlations, and having a shared factor structure (Loas et al., 1997). All three symptom rating scales tap visual and other forms of hallucinations, although a score of high or extreme frequency of experienced hallucinations will be based on the existence of auditory hallucinations. However, while the classic symptom scales are limited to the frequency and duration of hallucinations, other scales are more focused on the themes and contents of the voices and whether the voices cause distress and/or disruption to everyday life, like the revised Beliefs About Voices Questionnaire (BAVQ-R; Chadwick et al., 2000), and the Psychotic Symptoms Rating Scales (PSYRATS; Haddock et al., 1999).

The BAVQ-R is a self-report scale that does not need interpretation of the patient's response by a clinician to get a score, and contains simple statements, like 'the voice is controlling my life', that the patient answers by marking whether he/she agrees or disagrees. The BAVQ-R consists of 35 statements about experiences of 'hearing voices', the way in which the patient perceives the voice, the reactions of the patients to the voice and the beliefs held by the patient regarding the experience of 'hearing voices'. The scale is divided into three main sub-scales directly related to the contents of the voice that tap 'Malevolence' (six items: e.g., 'My voice is punishing me for something I have done'); 'Benevolence' (six items: e.g., 'My voice wants to protect me'); and 'Omnipotence' (six items: e.g., 'My voice is very powerful'). In addition, there are two other sub-scales measuring emotional and behavioral responses to the voices. The statements in BAVQ-R are phrased in a language that is common in diagnostic and therapeutic interviews and conversations. Responses to each item are scored on a four-point scale, ranging from 'Disagree' to 'Agree strongly' (see Chadwick et al., 2000 for further details). Summary of the above-mentioned symptom scales is given in Table 1.

\section{AUDITORY HALLUCINATIONS AS PERCEPTUAL MIS-REPRESENTATIONS}

In the following sections, an extended summary and review of the findings on auditory hallucinations that were originally presented as a regular Frontiers in Human Neuroscience article (Hugdahl et al., 2008) is presented. Here, an 
Table 1 | An overview of symptom scales described earlier, with names, acronyms, and a brief description of purpose and format of each scale.

\begin{tabular}{|c|c|c|c|c|}
\hline Name & Acronym & Purpose & Format & Reference \\
\hline $\begin{array}{l}\text { Brief Psychiatric } \\
\text { Rating Scale }\end{array}$ & BPRS & $\begin{array}{l}\text { Scale for assessing the } \\
\text { positive, negative, and } \\
\text { affective symptoms of individuals } \\
\text { who have psychotic disorders, } \\
\text { especially schizophrenia }\end{array}$ & $\begin{array}{l}\text { The BPRS consists of } 18^{*} \\
\text { symptom constructs that are } \\
\text { scored } 1-7 . \text { The symptom constructs cover } \\
\text { positive and negative symptoms of somatic } \\
\text { concern, anxiety and depression* }\end{array}$ & $\begin{array}{l}\text { Overall and } \\
\text { Gorham (1988) }\end{array}$ \\
\hline $\begin{array}{l}\text { Positive and } \\
\text { Negative } \\
\text { Syndrome } \\
\text { Scale }\end{array}$ & PANSS & $\begin{array}{l}\text { Scale for assessing } \\
\text { degree of positive, } \\
\text { negative symptoms, and general } \\
\text { psychopathology in } \\
\text { schizophrenia patients }\end{array}$ & $\begin{array}{l}\text { The PANSS consists of a } \\
\text { Positive scale ( } 7 \text { items), Negative scale } \\
\text { ( } 7 \text { items), and a General Psychopathology } \\
\text { scale (16 items). Each item is scored 1-7 }\end{array}$ & $\begin{array}{l}\text { Kay et al. } \\
\text { (1987) }\end{array}$ \\
\hline $\begin{array}{l}\text { Scale for Negative } \\
\text { Symptoms }\end{array}$ & SANS & $\begin{array}{l}\text { The SANS was } \\
\text { designed primarily as } \\
\text { descriptive instrument } \\
\text { that are useful for encoding negative } \\
\text { symptoms commonly observed } \\
\text { in psychiatric patients }\end{array}$ & $\begin{array}{l}\text { Clinical interview scale with } \\
\text { five dimensions: affective flattening } \\
\text { or blunting, alogia, avolition-apathy, } \\
\text { anhedonia-associability, attention, } \\
\text { with 3-7 items for each dimension. } \\
\text { Each item is scored 0-5 }\end{array}$ & $\begin{array}{l}\text { Andreasen } \\
\text { (1990) }\end{array}$ \\
\hline $\begin{array}{l}\text { Scale for Positive } \\
\text { Symptoms }\end{array}$ & SAPS & $\begin{array}{l}\text { The SAPS was designed } \\
\text { primarily as descriptive } \\
\text { instrument that are } \\
\text { useful for encoding positive } \\
\text { symptoms commonly observed in } \\
\text { psychiatric patients }\end{array}$ & $\begin{array}{l}\text { Clinical interview scale to be } \\
\text { used together with SANS. } \\
\text { Consists of five dimensions; } \\
\text { hallucinations, delusions, bizarre behaviour, } \\
\text { formal positive thought disorder, inappropriate } \\
\text { affect, ranging from } 1 \text { to } 13 \text { items per dimension. } \\
\text { Each item is scored } 0-5\end{array}$ & $\begin{array}{l}\text { Andreasen } \\
\text { (1990) }\end{array}$ \\
\hline $\begin{array}{l}\text { Beliefs About } \\
\text { Voices } \\
\text { Questionnaire - } \\
\text { Revised }\end{array}$ & BAVO-R & $\begin{array}{l}\text { The BAVQ-R measures, beliefs, } \\
\text { feelings and behaviour related to } \\
\text { auditory hallucinations in for } \\
\text { schizophrenia }\end{array}$ & $\begin{array}{l}\text { The BAVQ-R is a self-report questionnaire } \\
\text { with } 35 \text { items. Separate sub-scales } \\
\text { malevolence, benevolence and } \\
\text { omnipotence of the content of the voices }\end{array}$ & $\begin{array}{l}\text { Chadwick et al. } \\
\text { (2000) }\end{array}$ \\
\hline $\begin{array}{l}\text { Psychotic } \\
\text { Symptom Rating } \\
\text { Scale }\end{array}$ & PSYRATS & $\begin{array}{l}\text { Clinical Interview scale for } \\
\text { identification of auditory hallucinations } \\
\text { and delusions in psychotic patients }\end{array}$ & $\begin{array}{l}\text { The PSYRATS consists of two } \\
\text { dimensions; auditory hallucinations } \\
\text { and delusions, each with } 11 \\
6 \text { items, respectively, scored 0-4 }\end{array}$ & $\begin{array}{l}\text { Haddock et al. } \\
\text { (1999) }\end{array}$ \\
\hline
\end{tabular}

*In this article, an extended version of the BPRS scale with 24 items was used (Ventura et al., 1993).

\section{Dichotic listening}

It is a simple behavioral measure of brain laterality and hemispheric asymmetry.

Structural and functional MRI It provides information of anatomical and functional integrity of brain regions implicated in auditory hallucinations, respectively. extension of the original findings, and also new data collected after the original article was published, is given.

This study aims to advance the understanding of the cognitive and neuronal underpinnings of auditory hallucinations in schizophrenia. The methodology considered is to use an experimental neurocognitive approach using an auditory dichotic listening task (see the text below) together with structural and functional MRI in patients experiencing auditory hallucinations. The new findings revealed that hallucinating patients have problems in processing an external speech sound, particularly when experiencing ongoing hallucinations and also when instructed to use attention to better focus on the external stimulus. The phenomenon was paralleled in grey matter reduction in temporal lobe areas. The significance of the findings is discussed with regard to a neurocognitive model for auditory hallucinations, emphasizing perceptual mis-representations caused by neuronal abnormality in the temporal lobe areas.

Auditory hallucinations are also of interest from a psychological and neuroscience point of view as the patients may be as convinced that they hear a voice originating from outside of the person, as are healthy persons when they are actually spoken to. The difference between such patients and healthy individuals is, however, that there is no external acoustic source that could give rise to such a perception, a kind of 'inner speech' perception that is mistakenly equated by the patient with normal auditory perception.

As the cortical localization and the neural pathways for speech sound perception are quite well mapped out, not the least from recent functional neuroimaging studies (Binder and Price, 2001; Hickok and Poeppel, 2007; Hugdahl et al., 1999), it can be assumed that auditory hallucinations have their neuronal, or 'internal', origin in the same brain regions and networks that underlie normal 
speech perception (see also Hugdahl et al., 2008). Speech perception and production are lateralized to the left hemisphere, and localized to areas in the temporal and frontal lobes, respectively. Although more recent behavioral (e.g., Hugdahl, 2003) and brain imaging (Specht and Reul, 2003) studies have revealed a more complex and interactive pattern of speech localization in the brain, emphasizing ventral and dorsal areas (Hickok and Poeppel, 2007), most studies converge on the left temporal lobe as the core site for speech perception. Hence, if auditory hallucinations have neuronal origin in the same areas as normal speech perception, the left superior and middle temporal gyrus and superior temporal sulcus would be prime candidates, connecting ventrally with anterior parts of the temporal lobe and frontal regions and dorsally with parietal regions (see Figure 1).

The model presented in Figure 1 outlines a temporal-fronto-parietal neural circuit in which auditory hallucinations are considered as a bottom-up phenomenon that involuntarily attracts attention focus to the voices. Hence, the hypothesis is that auditory hallucinations are not kept in control, or inhibited, due to failure of prefrontal and anterior cingulate-mediated executive inhibition and response suppression. Such a hypothesis is supported by the findings of Rund et al. (2006), that patients with schizophrenia are impaired in executive and cognitive control functions that have a prefrontal localization. Rund et al. (2006) used an extensive neuropsychological test battery that tapped a wide range of cognitive functions, including memory recall and recognition, working memory, attention span and attention focus, executive functions, visuo-spatial function, and reaction time. The results showed that the schizophrenia group scored significantly lower as compared to a healthy control group and a group of depressed patients (included as a clinical comparison group).

On the basis of the findings of Hugdahl et al. (2008), it can be presumed that auditory hallucinations are caused by neuronal abnormality originating in the left temporal lobe speech areas and/ or neuronal grey matter pathology. Such a view is also in agreement with other studies on auditory verbal hallucinations, for e.g., 'mis-representations of inner experiences' (Heinks-Maldonado et al., 2007), 'mis-attribution of speech' (Allen et al., 2007), 'mis-attributed cognitions and inner speech' (Kinderman, 2007) or that auditory hallucinations may be 'competitions between auditory stimuli and competition for physiological resources in the temporal cortex' (Hubl et al., 2007). See also Woodruff (2004) for further discussion of the neuropsychology of auditory hallucinations.

A mis-representation hypothesis could be tested in several ways, using neuropsychological tests and functional brain imaging measures, like

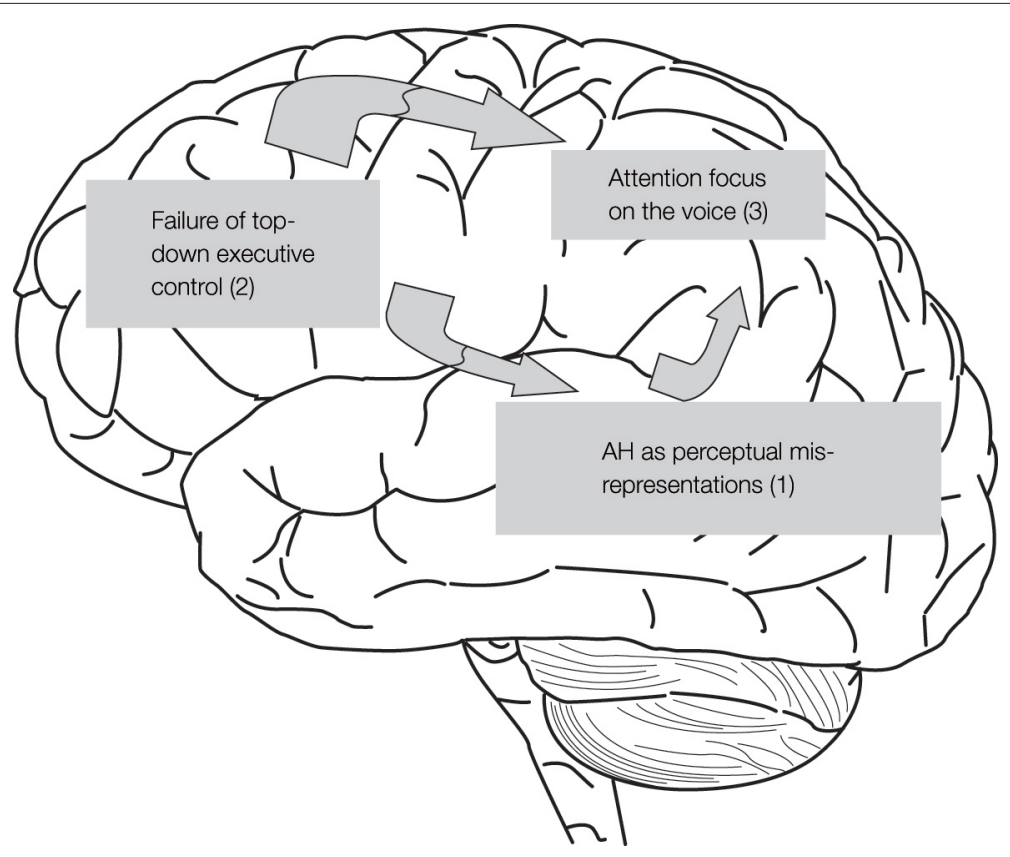

Figure 1 | Outline of a model for auditory hallucinations (AH) as perceptual mis-representations, parietal lobe attention enhancement and failure of prefrontal executive suppression control. The model emphasizes the involvement of the middle and superior temporal gyri (1) for the generation of $\mathrm{AH}$, prefrontal cortex (2) for top-down executive control, and parietal cortex (3) for attention focus. 


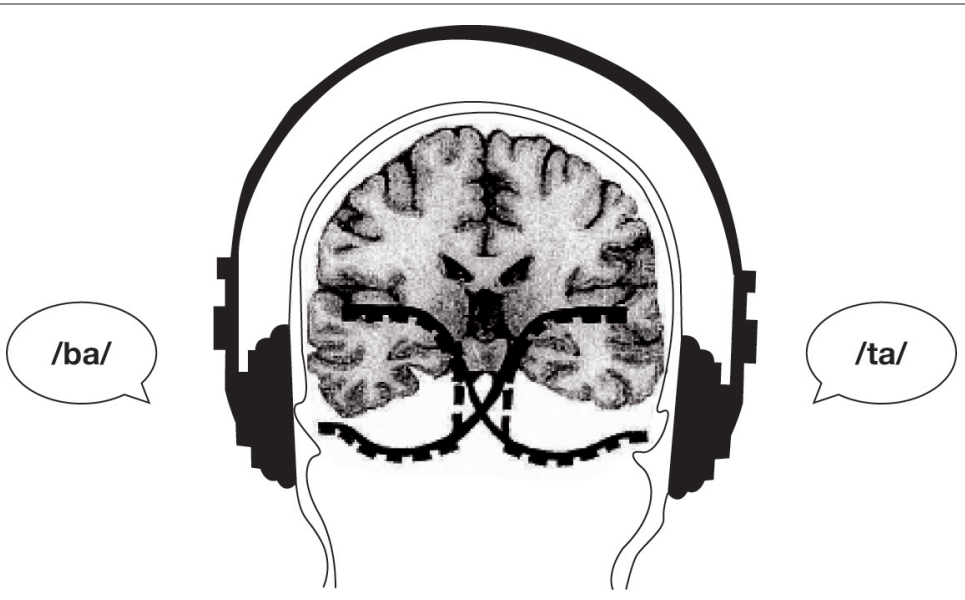

Figure 2 | Schematic illustration of the dichotic listening procedure in which two different consonant-vowel syllables are presented simultaneously, one syllable in the left ear and the other in the right ear. The task of the subject is to report the syllable heard/perceived on each trial. A trial with the syllable /ba/ presented in the left ear and the syllable/ta/ presented in the right ear is shown.

fMRI. In addition, pathology of grey matter tissue in the same areas in the brain would support a view of auditory hallucinations as abnormalities of neuronal activity, if it could be shown that hallucinating patients show reduced or altered brain tissue volume or density in the left temporal lobe. We describe an experimental neuropsychological approach, together with structural and functional MRI measures in the following sections.

\section{NEUROPSYCHOLOGICAL (DICHOTIC LISTENING) STUDIES}

In a neuropsychological study on auditory hallucinations, Green et al. (1994) used a simple dichotic listening procedure with pair-wise presentations of consonant-vowel (CV) syllables, and found that hallucinating patients failed to show a significant right ear advantage (REA), and instead showed a 50/50 distribution of answers for the right and left ears. The CV-syllables dichotic listening paradigm typically results in a REA in healthy individuals, i.e., more correct reports for the right ear stimulus of the dichotic pair, indicating a left temporal lobe speech perception locus (see Hugdahl, 2003; Hugdahl and Andersson, 1986; Pollmann et al., 2002). Dichotic listening means that two different stimuli are presented simultaneously, one stimulus in the right ear and the other in the left ear. The version of the dichotic listening procedure used in the laboratory consists of pair-wise presentations via headphones of simple CV syllables. The syllables are produced by pairing all possible combinations of the six stop-consonants $/ \mathrm{b} /, / \mathrm{d} /, / \mathrm{g} /, / \mathrm{p} /$, $/ \mathrm{t} /, / \mathrm{k} /$ and the vowel $/ \mathrm{a} /$. Hence, a single trial could be the presentation of the syllable /ba/ in the left ear and simultaneously the syllable/ta/ in the right ear (see Figure 2 for illustration of the dichotic listening procedure). The subject is not informed that each presentation consists of two different syllables, and is simply told to report on each trial 'the syllable they heard'. Due to the preponderance of the contralateral auditory pathways, a stimulus presented to the right ear will have direct access to the speech perception areas in the left temporal lobe, and at the same time block the ipsilateral left ear stimulus input to this area (Kimura, 1967). A typical finding in healthy individuals is the socalled REA which simply means that more correct items are reported for the right ear stimulus and would indicate that the hemisphere is dominant or specialized for speech perception and speech sound processing. A preference for reporting the syllable presented in the right ear is expected due to the phonological features of the syllable and as the speech sounds are perceptually processed in the left temporal lobe.

In addition to the standard procedure, the paradigm can also be used to study attentional and executive control functions, similar as seen in the Stroop-task (Lund-Johansen et al., 1996). The dichotic presentation mode produces a conflict situation when the subject is instructed to report only one syllable on each trial. Although a stimulus presented in e.g., the right ear has both ipsilateral and contralateral input to the temporal lobe processing areas, the contralateral pathways are more preponderant, and a dichotic presentation mode will further strengthen the input by blocking the ipsilateral input from the other ear (Kimura, 1967). The right ear syllable would have an initial unique access to the left hemisphere processing areas, thus causing a preferred right ear response 
from the subject. Hence, the CV syllables in a dichotic listening situation would be ideal for the study of left temporal lobe abnormality in auditory hallucinations, by allowing comparison of percentage of correct reports for the right ear and left ear syllables across trials. The failure of patients with schizophrenia to show a REA would then indicate a left temporal lobe functional deficit or impairment. The findings of Green et al. (1994) have later been reproduced in several laboratories (e.g., Conn and Posey, 2000; Hugdahl et al., 2008; Løberg et al., 2004; Rossell and Boundy, 2005).

\section{TRAINING IN THE DICHOTIC LISTENING SITUATION TO SUPPRESS THE VOICES}

An interesting extension of the dichotic listening method for revealing left temporal dysfunction in auditory hallucinations is the usage of a dichotic training procedure for improving the ability to ignore symptoms like hallucinations, and at the same time to improve other cognitive functions. However, the results have varied, Fiszdon et al. (2005) failed to show any training effects on auditory hallucinations, while Hatashita-Wong and Silverstein (2003) found indications of improvement. The idea behind the usage of a dichotic procedure for training to inhibit or ignore voice symptoms is that the dichotic situation gives rise to a very strong response tendency to report the right ear stimulus. If the patient can be trained in an experimental situation to suppress responding to the stimulus heard in the right ear, instead of shifting attention to the less dominant left ear stimulus, it can be generalized to suppress attention to the voices in real life.

\section{DICHOTIC LISTENING AND POSITIVE SYMPTOMS}

Frequent hallucinations almost always go together with other positive symptoms, like delusions, bizarre behaviors, and conceptual disorganization. A relationship between hallucinations and other positive symptoms was found by Løberg et al. (2006), using structural equation modeling to statistically model the association from performance on the dichotic listening task.

In this review, new data related to the question whether other positive symptoms also correlate negatively with magnitude of the REA in the dichotic listening test is presented. Table 2 gives correlations between dichotic listening laterality index scores and each of the 24 symptoms in the BPRS symptom questionnaire (Ventura et al., 1993) based on a sample of 100 patients with schizophrenia.

In Table 2, the symptoms showing a significant $(p<0.05)$ correlation with the dichotic listening laterality score were 'hallucinations' $(r=-0.34)$, 'unusual thought content' $(r=-0.30)$, and 'disorientation' $(-0.31)$. The dichotic listening laterality index is calculated from the formula $[($ REar $)-($ LEar $) /($ REar + LEar $)] \times 100$, where REar is the number of correct right ear responses and LEar is the number of correct left ear responses. A positive index score indicates a REA and a negative index score indicates a left ear advantage (LEA). The three significant correlations in Table 2 were all negative, with the largest correlation coefficient for the 'hallucination' symptom, which indicates that the higher the score on the BPRS 'hallucination' item the smaller

Table 2 | Correlation coefficients $(r)$ for the correlations between single BPRS symptoms and dichotic listening laterality index. Symptoms and correlation coefficients that are marked in red showed significant correlations with the dichotic listening laterality index. Explanations of BPRS symptoms and formula for calculation of dichotic listening laterality index score are given based on correct reports for the right and left ear stimulus, respectively (see the text above). The three standard positive symptoms are 'Hallucinations', 'Unusual Thought Content', and 'Bizarre Behavior'. The three standard negative symptoms are 'Conceptual Disorganization', 'Blunted Affect', and 'Emotional Withdrawal'.

$\begin{array}{lrlr}\text { Symptom } & \boldsymbol{r} & \text { Symptom } & \boldsymbol{r} \\ \text { Somatic concern } & -0.09 & \text { Self-neglect } & -0.16 \\ \text { Anxiety } & 0.12 & \text { Disorientation } & -0.31 \\ \text { Depression } & 0.04 & \text { Conceptual disorganization } & -0.01 \\ \text { Suicidality } & 0.05 & \text { Blunted affect } & -0.03 \\ \text { Guilt } & -0.19 & \text { Emotional withdrawal } & 0.02 \\ \text { Hostility } & -0.02 & \text { Motor retardation } & -0.06 \\ \text { Elevated mood } & 0.24 & \text { Tension } & -0.02 \\ \text { Grandiosity } & -0.19 & \text { Uncooperativeness } & 0.00 \\ \text { Suspiciousness } & -0.16 & \text { Excitement } & -0.06 \\ \text { Hallucinations } & -0.34 & \text { Distractibility } & -0.12 \\ \text { Unusual thought } & -0.30 & \text { Motor hyperactivity } & -0.10 \\ \text { Bizarre behavior } & -0.12 & \text { Mannerisms and postering } & 0.04\end{array}$


Non-hallucinating
patients (PANSS $\leq 3$ )

Hallucinating

patients (PANSS $>3$ )

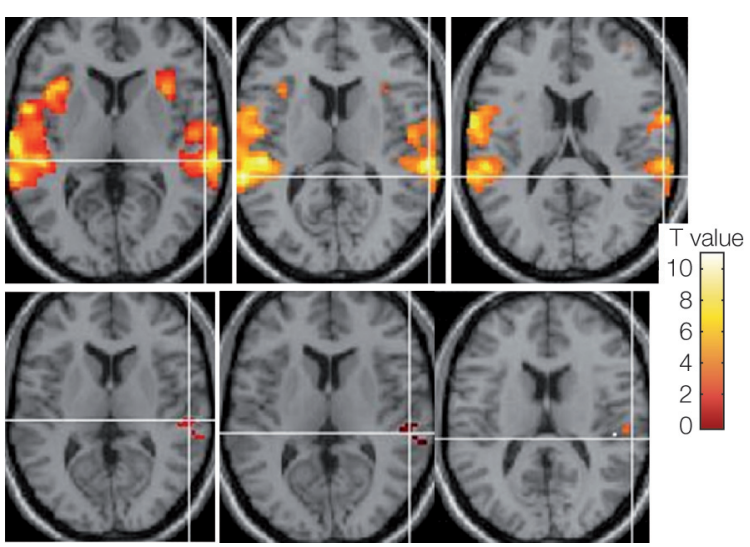

Figure 3 | fMRI activation data shown in three axial slices above the anterior-posterior commissure (AC-PC) midline in non-hallucinating (upper row) and hallucinating patients with schizophrenia when listening to dichotic presentations of speech sounds. Note the absence of significant activation in the left temporal lobe in the hallucinating patients $(p<0.001$, uncorrected)

the REA. Such an outcome would be predicted from a view that hallucinations originate in the left hemisphere speech perception areas, and is also consistent with the findings reported by Green et al. (1994) of absence of a REA in hallucinating patients. It is also interesting to note that the two other significant correlations were also negative for two other positive symptoms, 'unusual thought content' and 'disorientation'. Unusual thought content taps unusual, odd, strange, or bizarre thought content, and is also significantly correlative with hallucinations $(r=0.61$, $p<0.05)$. 'Disorientation' taps confusion and lack of proper associations for persons, place, or time, which covers a broader spectrum of excessive thoughts and behaviors, and is considered a general symptom rather than a positive symptom. In Table 2, positive, not negative symptoms, correlate negatively with performance on the dichotic listening task, indicating a specific left temporal lobe deficit.

\section{FUNCTIONAL NEUROIMAGING STUDIES}

Functional neuroimaging studies have measured, while placing in the MR scanner, either changes in blood flow or blood oxygen levels in patients during periods of hallucinations contrasted with periods of absence of hallucinations, or response to the presentation of external speech or other acoustic stimuli concordant with reports of hallucinations. Monitoring of neuronal activation and blood flow occurring during hallucinations in the absence of external stimuli have in general shown increased activation in temporal lobe areas (Dierks et al., 1999; Lennox et al., 2000; Shergill et al., 2004; Suzuki et al., 1993) and in other language-related areas, such as the thala- mus (Silbersweig et al., 1995), and Broca's area (McGuire et al., 1993; see also Weiss and Heckers, 1999 for review). In a mini-review, Sommer et al. (2003) also concluded that 'our results showed that language related areas in the left hemisphere were significantly more activated than the right-sided homotope regions' (p. 217).

Functional neuroimaging studies that have used externally presented speech stimuli, or provided instructions to generate inner speech perceptions, when in the scanner, have resulted in decreased activation in the same brain areas in hallucinating patients compared to healthy controls (Zhang et al., 2008), also when compared with non-hallucinating patients (McGuire et al., 1996). The results are supported by ERP-studies (e.g., Ford and Mathalon, 2005) showing that hallucinating patients have reduced or aberrant cortical activity to acoustic stimuli. Figure 3 shows fMRI activation data from the laboratory for patients experiencing frequent versus infrequent hallucinations. The subjects in this study as shown in Figure 3 were 17 patients with a DSM-IV or ICD-10 diagnosis of schizophrenia. In addition, the patients were screened with the PANSS symptom scale and grouped into two sub-groups based on their scores on the hallucination item in the PANSS.

Patients who received a score of higher than 3 ('moderate' to 'extreme' hallucinations) were grouped into a hallucination group while patients with a score of 3 or $<3$ ('absence' or 'mild' hallucinations) were grouped into a non-hallucination sub-group. All subjects were presented with the dichotic CV stimuli when placed in the scanner. As seen in Figure 3, the hallucinating patients failed to activate the speech areas in the upper 
posterior part of the temporal lobe, and in particular on the left side.

In addition, the patients shown in Figure 3 also failed to show activation in the anterior cingulate cortex when instructed to focus attention and report only from the left ear in the dichotic listening situation. The condition, labeled as 'forcedleft attention' by Hugdahl and Andersson (1986), involves a bottom-up and top-down processing conflict in the sense that the bottom-up stimulus-driven effect pushes for a right ear response while the top-down instruction-driven effect pushes for a left ear response (cf. Thomsen et al., 2004).

Figure 4 shows that the same patients failed to activate what could be called a generalized effort network when contrasting the images during the forced-left instruction condition with the images obtained during the forced-right instruction condition. A generalized effort network would be activated whenever demands for recruitment of higher-order cognitive functions, like attention, working memory and executive, or control functions, are called for and would show activations in prefrontal cortex, anterior cingulate and inferior parietal cortex. A generalized effort network would be activated orthogonal to the default mode or resting state network as suggested by Fox and Raichle (2007). Such a state is activated in the absence of explicit tasks or instructions and contains the medial frontal gyrus, middle temporal gyrus, pre-cuneus, and posterior cingulate cortex. As seen in Figure 4, hallucinating patients fail in the recruitment of higher cognitive functions necessary for adequate top-down suppression and executive control. It is proposed that cognitive impairments in schizophrenia, including inhibitory control of hallucinations in addition to a temporal lobe abnormality, may involve failure of down-regulation of a resting-state network and corresponding up-regulation of an effort network, thus upsetting the normal functioning of cognitive control mechanisms.

\section{RESOLVING THE FUNCTIONAL NEUROIMAGING CONTRADICTION}

The apparent contradicting findings with regard to functional neuroimaging, with both increased and decreased activation in hallucinating patients, can now be resolved within a single model. Increased activation in the absence of external stimuli during hallucinations would be due to abnormal or spontaneous neuronal activity. Failure of a response to external speech or acoustic stimulus would be due to the neurons that are already engaged by an internally generated activity, thus showing a kind of neuronal refractory effect to external stimuli.

The fact that different mechanisms may be involved for left temporal lobe activation during spontaneous hallucinations and the absence of activation when hallucinating patients are required to process an external speech sound is supported by the findings of McGuire et al. (1996) who instructed their patients to imagine hearing a person speaking to them, which would be a kind of 'external' stimulus, although internally

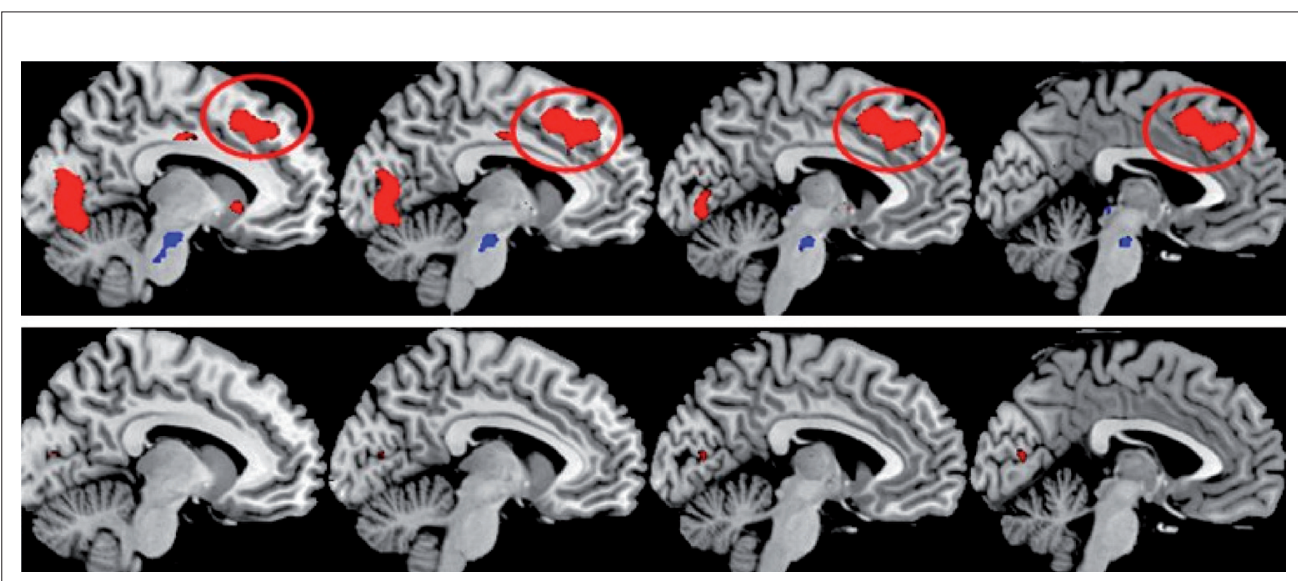

Figure 4 | fMRI activation data in a healthy control group (upper row) and patients with schizophrenia (lower row) when the images acquired during instructions to attend to the left ear stimulus were in contrast with the images acquired during instructions to attend to the right ear stimulus. The sagittal displays are from $-9 \mathrm{~mm}$ (furthest left display) to $-3 \mathrm{~mm}$ (furthest right display) from the midline, in the left hemisphere. The red ovals highlight the activation in the anterior cingulate cortex, which is a part of a proposed generalized effort network. Other areas included in the generalized effort network are prefrontal and parietal areas (not shown in the figure) as well as occipital areas ( $p<0.5$, corrected). 
generated. They concluded that 'when imagining sentences spoken in another person's voice, which entails the monitoring of inner speech, hallucinators showed reduced activation in the left middle temporal gyrus and the rostral supplementary motor area, regions which were strongly activated by both normal subjects and non-hallucinators' (p. 148).

It is therefore suggested that auditory hallucinations elicit spontaneous neuronal activation in the left temporal lobe that blocks reception of speech stimuli when presented e.g., in a dichotic mode, which allows direct probing of left temporal lobe functional integrity. Such an explanation could then also accommodate the findings of increased neuronal activation in hallucinating patients in the absence of external auditory stimuli.

\section{STRUCTURAL NEUROIMAGING STUDIES}

Structural MRI studies have confirmed abnormality in the speech areas in the left temporal lobe in patients with schizophrenia, with reduced grey matter density and volume on the left side compared to the right side (e.g., Barta et al., 1990; Kubicki et al., 2002; Lawrie and Abukmeil 1998; Neckelmann et al., 2006). In a recent meta-analysis of 17 studies, Williams (2008) showed that reduction in grey matter volume and density in the left superior temporal gyrus in patients with schizophrenia is the most consistently reported brain structural abnormality. More than $80 \%$ of the analyzed studies reported this abnormality.

Interestingly, the left temporal lobe abnormality was also seen in first-episode patients, which would support the above-mentioned argument that positive symptoms related to left hemisphere abnormality may be a cause, rather than a consequence, of the disorder. A common finding is that such morphological abnormalities correlate with frequency and duration of auditory hallucinations (e.g., Milev et al., 2003; Neckelmann et al., 2006; Shapleske et al., 2002; Shin et al., 2005). Although different studies show great variability with regard to the methods used (e.g., whole brain versus region-of-interest approaches) and results, a majority of the studies reviewed by Williams (2008) have reported abnormalities of grey matter density and volume in fronto-temporal areas. This has also been shown for cortical thickness, with thinning of the cortical mantle in the left superior temporal gyrus and in prefrontal areas, including the inferior and middle frontal gyrus (Nesvåg et al., 2008).

Discussing the mechanisms underlying reduction in grey matter density or volume, two hypotheses can be put forward. The first hypothesis is that reduction in grey matter density is due to reduction in neuron size across the affected areas. This was addressed in a recent study by Pergolizzi et al. (2008) who did not find evidence for smaller neuron size in the left superior temporal gyrus, including the planum temporale, in patients with schizophrenia. The second hypothesis is that reduction of the cortical mantle thickness means compressing neuron density with less widely spaced cellular columns on the left side, which in turn could lead to decreased connectivity per neuron.

Using a voxel-based morphometry (VBM) approach, García-Martí et al. (2008) reported reduction in grey matter tissue concentration in the left superior temporal gyrus and amygdala, and bilaterally in the insula in hallucinating patients. In addition, there were also significant differences between patients having reduction of grey matter density and controls in the left inferior frontal gyrus which would affect Broca's area (cf. McGuire et al., 1993). The study of GarcíaMartí et al. (2008) used the PSYRATS scale for further quantification of characteristic features of auditory hallucinations beyond frequency and duration. The usage of more direct symptom scales like the PSYRATS and BAVQ-R could yield more important information regarding themes and content of the hallucinations that may be uniquely correlated with neuropsychological and brain functional and structural measures.

In the Neckelmann et al. (2006) study, VBM was used for comparison of grey matter density in schizophrenia patients and healthy controls. An additional analysis also involved splitting the patients into a sub-group that revealed a REA on the dichotic listening test and a sub-group that failed. The Neckelmann et al. (2006) study provided data for the hypothesis proposed by Green et al. (1994), that the failure to show a REA in the dichotic listening test could have a neuronal origin in the upper posterior region of the left temporal lobe. Data that were not previously presented from the Neckelmann et al. (2006) study are shown in Figure 5. According to these data, the sub-group of patients who failed to show a REA (left-hand panel) were the patients who showed significant reductions in grey matter density, particularly in the superior gyrus of the left temporal lobe.

\section{FURTHER QUESTIONS STATE OR TRAIT EFFECT?}

Løberg et al. (2004) posed a question whether the effect of hallucinations on dichotic listening performance is a state or trait effect, by comparing patients having ongoing hallucinations with patients having previous hallucinations. The patient sub-groups (and healthy control group) underwent the same dichotic 


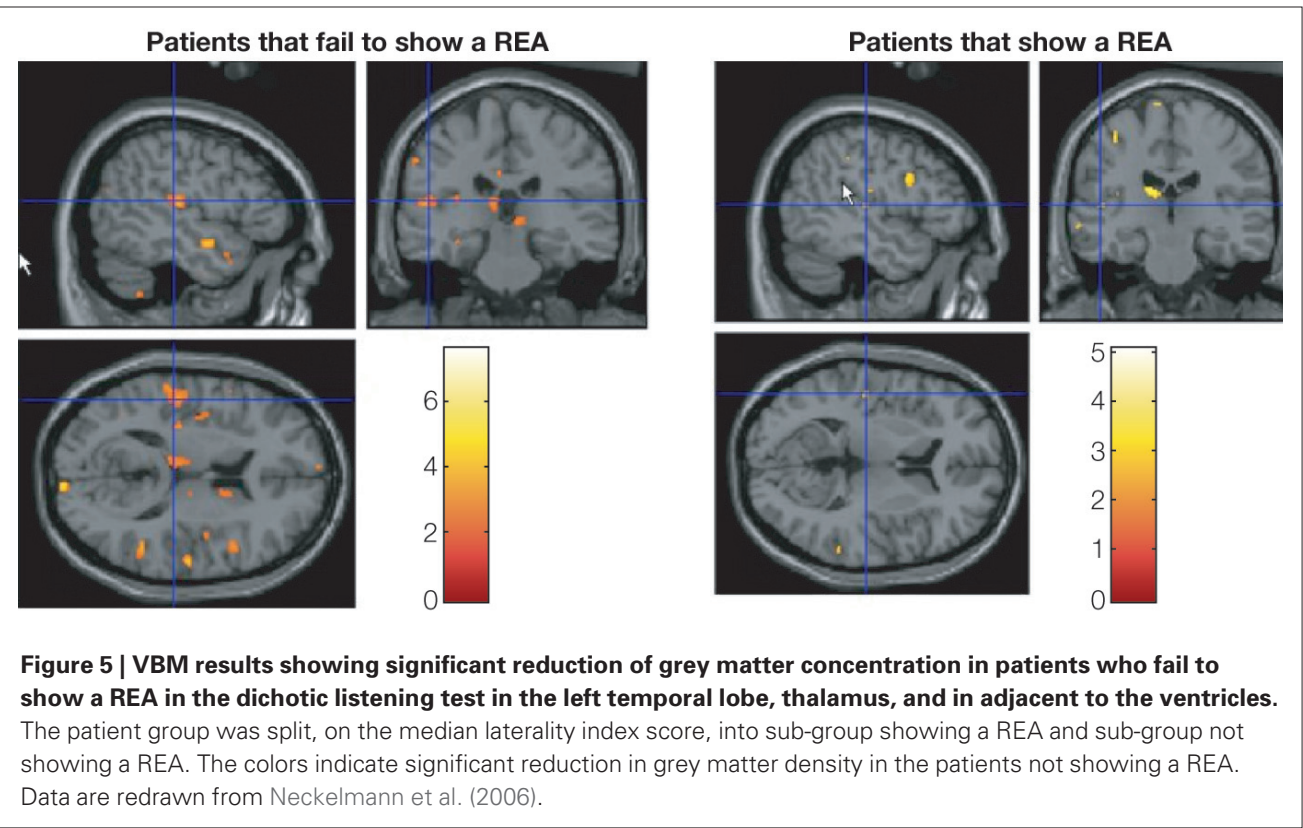

listening protocol as the patients in the Green et al. (1994) study. The patient group was split into the two sub-groups by using scores from the PANSS hallucination item. Previous experience with hallucinations and the auditory quality was validated through a questionnaire specifically designed for the study, in addition to the patient's records and SCID interviews. The ongoing hallucination group showed a significantly smaller REA compared to the healthy control group and the patients with previous hallucinations. The reduced REA in the ongoing hallucinations subgroup indicates that state-factors may be more important than trait-factors when it comes to the ability to process an external speech sound presented to the left hemisphere.

\section{DOES FREQUENCY OF HALLUCINATIONS MATTER?}

It could be hypothesized that if auditory hallucinations are related to internally generated distorted speech perception originating in speech areas in the left temporal lobe, then the reduction of the REA in the dichotic listening situation would be greater for patients experiencing more frequent hallucinations than for patients experiencing few or no hallucinations. As a follow-up and replication of previous results, we have investigated the results by comparing patients having few or no hallucinations with patients having frequent hallucinations. The two patient groups were also compared with a group of healthy control subjects, using PANSS scores to split the patients into sub-groups. An analysis of variance showed a failure of a significant REA in the 'frequent hallucinations' patient group, while the 'no hallucinations' patient group and the healthy control group both had a significant REA (see Hugdahl et al., 2008 for further details).

\section{SYNAPTIC MEDIATION?}

We are currently investigating a glutamate hypothesis (see Hugdahl et al., 2008) for auditory hallucinations by administrating the drug memantine while healthy subjects are undergoing the dichotic listening test in the MR scanner. Memantine is a drug used in the treatment of Alzheimer's disease and blocks the action of glutamate at the synaptic level, i.e., it acts as a NMDA receptor antagonist. The design was a cross-over design so that all subjects also participated when not on memantine.

Preliminary results have shown that memantine reduces activation in the left superior temporal gyrus, possibly by inhibiting the excitatory action of glutamate at the receptor site, while prefrontal activation was increased when comparing the drug-naive and memantine conditions.

A glutamate hypothesis has been proposed for schizophrenia (see Carlsson et al., 1999; Kehrer et al., 2008; Olney and Farber, 1995), suggesting that glutamate function in addition to dopamine function may be altered in patients with schizophrenia. The glutamate hypothesis proposes a hypo-functional glutamate system. Although reasoning by analogy from results on healthy individuals, it is suggested that glutamatergic transmission may be altered in auditory hallucinations, which may trigger the experience of 'hearing voices' (cf. Bartha et al., 1999).
It mediates auditory hallucinations a the receptor level. 


\section{IS THE ABNORMALITY UNIOUELY LOCALIZED TO THE LEFT HEMISPHERE?}

A question that remains unanswered is whether the observed structural and functional abnormalities related to left hemisphere anatomy and neuronal activation are unique for the left hemisphere and left temporal lobe, and what is the role played by the right hemisphere.

Pollmann et al. (2002) found that the right hemisphere does not process dichotically presented CV syllables in healthy individuals, using a stimulus task similar to the one used in the studies described earlier (e.g., Green et al., 1994; Hugdahl et al., 2008). Hence, it is unlikely that right temporal lobe abnormality would contribute to the failure of showing a REA in the schizophrenia patients. However, Figure 3 also shows a reduction of activation in the right hemisphere in the hallucinating patients. It is therefore at present not clear whether such a condition is related to a functional impairment in processing externally presented speech sounds in the patients, or whether it is unrelated to functional impairment. In her meta-analysis of structural abnormality in schizophrenia, Williams (2008) did not find evidence for a right temporal lobe abnormality, thus supporting a view that the grey matter abnormality is uniquely related to the left hemisphere. However, it still remains to be seen whether this is the cause or the consequence of auditory hallucinations in schizophrenia.

\section{SUMMARY AND CONCLUSIONS}

The research on which the present review is based (Hugdahl et al., 2008) have shown that patients with schizophrenia who experience frequent auditory hallucinations show reduced grey matter density and neuronal activation when measured with VBM analysis from structural MR images. Similarly, analysis fMRI images to dichotic presentations of CV syllables have shown reduced activation, compared to healthy controls in the left temporal lobe and in anterior cingulated cortex. The brain structural and functional abnormalities seem to converge in the superior gyrus of the left temporal lobe.

In addition, the same patients show reduced activation in the dorsal portion of the anterior cingulate cortex, that could be related to inability to volitionally inhibit the hallucinatory impulses. The failure of activation in the anterior cingulate cortex could be a neuronal correlate of inability to inhibit internally generated 'voices' in the form of speech mis-representations, further enhanced by focusing of attention on the voices once they are elicited, as part of a dysfunctional fronto-parietal neuronal network.

\section{ACKNOWLEDGMENTS}

The authors wish to acknowledge the contributions of Heidi van Wageningen, Vidar M. Steen, and Hugo A. Jørgensen for data analysis and commentaries on the research presented in the focused review. The research reported was funded by grants to Kenneth Hugdahl from the Research Council of Norway (NEVRONOR), the Health Authority of Western Norway, The Haukeland University Hospital Strategic Research Programme, and the Nyquist Legat, Bergen, Norway.

\section{REFERENCES}

Allen, P., Amaro, E., Fu, C. F., Williams, S. C. R., Brammer, M. J., Johns, L. C., and McGuire, P. K. (2007). Neural correlates of the misattribution of speech in schizophrenia. Br. J. Psychiatr. 190, 162-169.

American Psychiatric Association (1994). Diagnostic Manual for Mental Disorders (DSM-IV). Washington, DC, American Psychiatric Association.

Andreasen, N. C. (1990). Methods for assessing positive and negative symptoms. Mod. Probl. Pharmacopsychiatry 24, 73-88.

Barta, P. E., Pearlson, G. D., Powers, R. E., Richards, B. S., and Tune, L. E. (1990). Auditoryhallucinations and smallersuperior temporal gyral volume in schizophrenia. Am. J. Psychiatry, 147, 1457-1462.

Bartha, R., Al-Semaan, Y. M., Williamson, P. C., Drost, D. J., Malla, A. K., Carr, T. J., Densmore, M. Canaran, G., and Neufeld, W. R. J. (1999).A short echo proton magnetic resonance spectroscopy study of the left mesial-temporal lobe in first-onset schizophrenic patients. Biol. Psychiatry 45, 1403-1411.

Binder, J., and Price, C. J. (2001). Functional neuroimaging of language. In Handbook of Functional Neuroimaging of Cognition, A. Kingstone, ed. (Cambridge, MIT Press), pp. 187-250.

Carlsson,A.,Waters, N., and Carlsson, M. L. (1999). Neurotransmitter interactions in schizophrenia-therapeutic implications. Eur. Arch. Psychiatry Clin. Neurosci. 249, 37-43.

Chadwick, P., Lees, S., and Birchwood, M. (2000). The revised beliefs about voices questionnaire (BAVQ-R). Br. J. Psychiatry 177, 229-232.

Conn, R., and Posey, T. B. (2000). Dichotic listening in college students who report auditory hallucinations. J. Abnorm. Psychol. 109, 546-549.

Dierks, T., Linden, D. E. J., Jandl, M., Formisano, E., Goebel, R., Lanfermann, H., and Singer, W. (1999). Activation of Heschl's gyrus during auditory hallucinations. Neuron 22, 615-621.

Fiszdon, J.M., Whelahan, H., Bryson, G. J. Wexler, B. E., and Bell, M. D. (2005). Cognitive training of verbal memory using a dichotic listening paradigm: impact on symptoms and cognition. Acta Psychiatr. Scand. 117, 187-193.

Ford, J. M., and Mathalon, D. H. (2005) Corollary discharge dysfunction in schizophrenia: can it explain auditory hallucinations? Int. J. Psychophysiol. 58, 179-189.

Fox, M., and Raichle, M. (2007). Spontaneous fluctuations in brain activity observed with functional magnetic resonance imaging. Nat. Neurosci. Rev. 8, 700-711.

García-Martí, G., Aguilar, E. J., Lull, J. L., Martí-Bonmatí, L., Escartí, M. J., Manjón, J. V., Moratal, D., Robles, M., and Sanjuán, J. (2008). Schizophrenia with auditory hallucinations: a voxel-based morphometry study. Prog. Neuropsychopharmacol. Biol. Psychiatry 32, 72-80.
Green, M. F., Hugdahl, K., and Mitchell, S. (1994). Dichotic listenig during auditory hallucinations in schizophrenia. Am. J. Psychiatry 151, 357-362.

Haddock, G., McCarron, J., Tarrier, N., and Faragher, E. B. (1999). Scales to measure dimensions of hallucinations and delusions: the psychotic symptom rating scales (PSYRATS). Psychol. Med. 29, 879-889.

Hatashita-Wong, M., and Silverstein, S. M. (2003). Coping with voices: selective attention training for persistent auditory hallucinations in treatment refractory schizophrenia. Psychiatry 66, 255-261.

Heinks-Maldonado, T., Mathalon, D. H., Houde,J.H.,Gray,M.,Faustman, W. O., and Ford, J. M. (2007). Relationship of imprecise corollary discharge in schizophrenia to auditory hallucinations. Arch. Gen. Psychiatr. 64, 286-296.

Hickok, G., and Poeppel, D. (2007). The cortical organization of speech processing. Nat. Rev. Neurosci. 8, 393-402. 
Hubl, D., Koening, T., Strik, W. K., Melie Garcia, L., and Dierks, T. (2007). Competition for neuronal resources: how hallucinations make themselves heard. Br. J. Psychiatry 190, 57-62.

Hugdahl, K. (2003). Dichotic listening in the study of auditory laterality. In The Asymmetrical Brain, K. Hugdahl and R. J. Davidson, eds (Cambridge, MIT Press), pp. 441-478.

Hugdahl, K., and Andersson, L. (1986). The 'forced-attention paradigm' in DL to CV-syllables: a comparison between adults and children. Cortex 22, 417-432.

Hugdahl, K., Brønnick, K., Law, I., Kyllingsbæk, S., and Paulson, O. B. (1999). Brain activation during dichotic presentations of consonantvowel and musical instruments stimuli: a 15O-PET study. Neuropsychologia 37, 431-440.

Hugdahl, K., Løberg, E.-M., Specht, K., Steen, V. M., Wageningen, H., and Jørgensen, H. A. (2008). Auditory hallucinations in schizophrenia: the role of cognitive, brain structural and genetic disturbances in the left temporal lobe. Front. Hum. Neurosci. 1, 6 . doi:10.3389/neuro.09/006.2007.

Kay, S. R., Fiszbein, A., and Opler, L. A. (1987). The positive and negative syndrome scale (PANSS) for schizophrenia. Schizophr. Bull. 13, 261-276.

Kehrer, C., Mashiashvili, N., Dougladze, T., and Gloveli, T. (2008). Altered excitatory-inhibitory balance in the NMDA-hypofunction model of schizophrenia. Front. Mol. Neurosci. 1, 6. Epub April 8, doi: 10.3389/ neuro.02.006.2008

Kimura, D. (1967). Functional asymmetry of the brain in dichotic listening. Cortex 3, 163-168.

Kinderman, P. (2007). Auditory hallucinations. J. Norw. Psychol. Assoc. 44, 547-554.

Krabbendam, L., and van Os, J. (2005). Schizophrenia and urbanicity: a major environmental influence conditional on genetic risk. Schizophr. Bull. 31, 795-799.

Kubicki,M.,Shenton,M.E.,Salisbury, D. F., Hirayasu, Y., Kasai, K., Kikinis, R., Jolesz, F. A., and McCarley, R. W. (2002). Voxel-based morphometric analysis of gray matter in first episode schizophrenia. Neuroimage 17, 1711-1719.

Lawrie, S. M., and Abukmeil, S. S. (1998). Brain abnormality in schizophrenia. A systematic and quantitative review of volumetric magnetic resonance imaging studies. Br. J. Psychiatry 172, 110-120.

Lennox, B. R., Park, S. B., Medley, I., Morris, P. G., and Jones, P. B. (2000). The functional anatomy of audi- tory hallucinations in schizophrenia. Psychiatry Res. 100, 13-20.

Loas, G., Noisette, C., Legrand, A., and Delahousse, J. (1997). A fourdimensional model of chronic schizophrenia based on the factorial structure of the positive and negative syndrome scale (PANSS). A study of a group of 153 chronic schizophrenic patients and comparison with the factorial structure of the BPRS. Encephale 23, 10-18.

Løberg, E.M., Jorgensen,H.A., Green, M. F., Rund, B. R., Lund, A., Diseth, Å., Øie, M., and Hugdahl, K. (2006). Positive symptoms and duration of illness predict functional laterality and attention modulation in schizophrenia. Acta Psychiatr. Scand. 113, 322-331.

Løberg, E. M., Jørgensen, H. A., and Hugdahl, K. (2004). DL in schizophrenic patients: effects of previous vs. ongoing auditory hallucinations. Psychiatry Res. 128, 167-174.

Lund-Johansen, M., Hugdahl, K., and Wester, K. (1996). Cognitive function in patients with Parkinson's disease undergoing stereotaxic thalamotomy. J. Neurol. Neurosurg. Psychiatr. 60, 564-571.

McGuire, P. K., Murray, R. M., and Shah, M. S. (1993). Increased blood flow in Broca's area during auditory hallucinations in schizophrenia. Lancet 342, 703-706.

McGuire, P. K., Silbersweig, D. A., Wright, I., Murray, R. M., Frackowiak, R. S., and Frith, C. D. (1996). The neural correlates of inner speech and auditory verbal imagery in schizophrenia: relationship to auditory verbal hallucinations. $\mathrm{Br}$. J. Psychiatry 169, 148-159.

Milev, P., Ho, B. C., Arndt, S., Nopoulos, P., and Andreasen, N. C. (2003). Initial magnetic resonance imaging volumetric brain measurements and outcome in schizophrenia: a prospective longitudinal study with 5-year follow-up. Biol. Psychiatry 54, 608-615.

Neckelmann, G., Specht, K., Lund, A., Ersland, L., Smievoll, A. I., and Hugdahl, K. (2006). MR morphometry analaysis of grey matter density reducution in schizophrenia: interactions with hallucinations. Int. J. Neurosci. 116, 9-23.

Nesvåg, R., Lawyer, G., Varnäs, K., Fjell, A. M., Walhovs, K. B., Frigessi, A., Jönsson, E. G., and Agartz, I. (2008). Regional thinning of the ecrebral cortex in schizophrenia: effects of diagnosis, age and antipsychotic medication. Schizophr. Res. 98, 16-28.

Olney, J. W., and Farber, N. B. (1995). Glutamate receptor dysfunction and schizophrenia. Arch. Gen. Psychiatry 52, 998-1007.
Overall,J.E., and Gorham,D.R.(1988). The brief psychiatric rating scale (BPRS): recent developments in ascertainment and scaling. Psychopharmacol. Bull.24, 97-99.

Pergolizzi, D., Dwork, A. J., Rosoklija, G. Dias, E. C., Javitt, D. C., and Smiley, J. F. (2008). Hemispheric comparison of neuron size in schiophrenia auditory cortex. Poster presented at the Society for Neuroscience annual meeting, Washington DC, November 18.

Pollmann, S., Maertens, M., von Cramon, D. Y., Lepsien, J., and Hugdahl, K. (2002). Dichotic listening in patients with splenial and nonsplenial callosal lesions. Neuropsychology 16, 56-64.

Rossell, S. L., and Boundy, C. L. (2005) Are auditory-verbal hallucinations associated with auditory affective processing deficits. Schizophr. Res. 78, 95-106.

Rund, N. R., Sundet, K., Asbjørnsen, A. Egeland, J., landrø, N. I., Lund, A. Roness,A.,Stordal,K.I.,andHugdahl, K (2006). Neuropsychological testprofiles in schizophrenia and non-psychotic depression. Acta Psychiatr. Scand. 113, 350-359.

Shapleske, J., Rossell, S. L., Chitnis, X. A., Suckling, J., Simmons, A., Bullmore, E. T., and Woodrudff, P. (2002). A computational morphometric MRI study of schizophrenia: effects of hallucinations. Cereb. Cortex 12, 1331-1341.

Shergill, S. S., Brammer, M. J., Amaro, E., Williams, S. C. R., Murray, R. M., and McGure, P. K. (2004). Temporal course of auditory hallucinations. $\mathrm{Br}$. J. Psychiatry 185, 516-517.

Shergill, S. S., Murray, R. M., and McGuire, P. K. (1998). Auditory hallucinations: a review of psychological treatments. Schizophr. Res. 32, 37-50.

Shin, S. E., Lee, J.-S., Kang, M.-H. Kim, C- E., Bae, J.-N., and Jung, G. (2005). Segmented volumes of cerebrum and cerebellum in first episode schizophrenia with auditory hallucinations. Psychiatry Res. 138 , 33-42.

Silbersweig, D. A., Stern, E., Frith, C. Cahill, C., Holmes, A., Grootonk, S. Seaward, J., McKenna, P., Chua, S. E. Schnorr,L., Jones, T., and Frackowiak, R J.S. (1995).A functional neuroanatomy study of hallucinations in schizophrenia. Nature 378, 176-179.

Sommer, I., Aleman, A., and Kahn, R. S. (2003). Left with the voices or hearing right? Lateralization of auditory verbal hallucinations in schizophrenia. J. Psychiatry Neurosci. 28, 217-218.

Specht, K., and Reul, J. (2003). Functional segregation of the temporal lobes into highly differentiated subsystems for auditory perception: an auditory rapid event-related fMRI-task. Neuroimage 20, 1944-1954.

Suzuki, M., Yasa, S., Minabe, Y., Murata, M., and Kurachi, M. (1993). Left superior temporal blood flow increases in schizophrenic and schizophreniform patients with auditory hallucinations: a longitudinal case study using 123I-IMP SPECT. Eur. Arch. Psychiatry Clin. Neurosci. 242, 257-261.

Thomsen, T., Rimol, L. M., Ersland, L., and Hugdahl, K. (2004). Dichotic listening reveals functional specificity in prefrontal cortex: an fMRI study. Neuroimage 21, 211-218.

Tien, A. (1991). Distributions of hallucinations in the population. Soc. Psychiatry Psychiatr. Epidemiol. 26, 287-292.

Ventura, J., Green, M. F., Shaner, A., and Liberman, R. P. (1993). Training and quality assurance with the Brief Psychiatric Rating Scale: 'The drift buster'. Int. J. Methods Psychiatr. Res. 3, 221-244.

Weiss, A. P., and Heckers, S. (1999). Neuroimaging of hallucinations: a review of the literature. Psychiatry Res. 92, 61-74.

Williams, L. M. (2008). Voxel based morphometry in schizophrenia: implications for neurodevelopmental connectivity models and cognition and affect. Expert Rev. Neurother. 8, 1029-1036.

Woodruff, P.W. R. (2004).Auditory hallucinations: insights and questions from neuroimaging. Cogn. Neuropsychiatry 9, 73-92.

Zhang, Z., Shi, J., Yuan, Y., Hao, G., Yao, Z., and Chen, N. (2008). Relationship of auditory verbal hallucinations with cerebral asymmetry in patients with schizophrenia: an event-related fMRI study. J. Psychiatr. Res. 42, 477-486.

Conflict of Interest Statement: The authors declare that the research was conducted without any commercial or financial relationships that could be construed as a potential conflict of interest.

Received: 06 October 2008; paper pending published: 05 November 2008; accepted: 08 January 2009; published: 01 May 2009. Citation: Front. Neurosci. (2009) 3,1: 34-45. doi:10.3389/neuro.01.001.2009

Copyright (c) 2009 Hugdahl, Løberg and Nygård. This is an open-access article subject to an exclusive license agreement between the authors and the Frontiers Research Foundation, which permits unrestricted use, distribution, and reproduction in any medium, provided the original authors and source are credited. 